\title{
ANTISENSE PROTEIN KINASE A RI $\alpha$-INDUCED TUMOR REVERSION: PORTRAIT OF A MICROARRAY
}

\author{
YOON S. CHO-CHUNG \\ National Cancer Institute, National Institutes of Health, Bethesda MD \\ 20892-1750, USA
}

The use of oligonucleotides to control gene expression has long fascinated researchers in basic science and medicine. Antisense oligonucleotides can selectively block disease-causing genes through the specificity of the WatsonCrick base-pairing mechanism of action. Genome sequencing has characterized thousands of proteins, and high-throughput gene-expression profiling [1] is a powerful tool for probing the cellular consequences of perturbing protein function.

We examined the genomic effects of antisense inhibition of protein kinase A RI $\alpha$ expression in tumor cells [2]. This study revealed that the expression of many genes was altered, producing expression signatures that created a molecular portrait of reverted tumor cells and allowed identification of subtle or unexpected effects of target-gene inhibition. The antisense affects one cluster, or signature, of genes involved in proliferation and another involved in differentiation [2]. The antisense downregulates proliferation-transformation signature genes and upregulates differentiation-reverse-transformation signature genes. Strikingly, expression signatures induced by exogenously supplied antisense oligonucleotides mirror those induced by the antisense gene that is endogenously overexpressed. In in vivo tumor models, the specificity of antisense effects on gene expression signatures was verified using three antisense oligonucleotides that differed in sequence or chemical modification.

Microarray technology has expanded the horizons of antisense technology; it advances the promise of antisense beyond a single target gene to the whole cell and the whole organism. For the first time, cDNA microarrays have revealed that antisense PKA RI $\alpha$ can modulate a wide set of genes related to cell proliferation versus differentiation in a sequence-specific manner in tumor cells. These expression signatures were quiescent and unaltered in the host livers of antisense-treated animals [2]. This clearly indicates that distinct cAMP signaling pathways regulate growth for normal and cancer cells. RI $\alpha$ antisense induces molecular differentiation signatures in cancer cells in a sequence-specific manner, leading to induction of a new reverted phenotype.

Unlike conventional chemotherapy regimens, that depend on a given drug's maximum tolerated dose for optimal tumor-cell kill, treatment regimens involving antisense oligonucleotides rely on an optimal biological dose. The ultimate goal for therapeutic oligonucleotides is their use as long-term biological gene modulators with minimal or no toxicity. 
Along with recent rapid advances in oligonucleotide technologies, including new chemical and biological understanding of PNA, LNA, morpholino, 2-0-alkyl RNA, second-generation antisense DNAs, and interfering RNAs, oligonucleotide-based gene silencing now offers an exciting possibility for treating and preventing cancer and other diseases [3-5].

\section{REFERENCES}

1. Schena, M., Shalon, D., Davis, R.W. and Brown, P.O. Quantitative monitoring of gene expression patterns with a complementary DNA microarray. Science 270 (1995) 467-470.

2. Cho, Y.S., Kim, M.K., Cheadle, C., Neary, C., Becker, K.G. and ChoChung, Y.S. Antisense DNAs as multisite genomic modulators identified by DNA microarray. Proc. Natl. Acad. Sci. USA 98 (2001) 9819-9823.

3. Gewirtz, A.M.J. Oligonucleotide therapeutics: A step forward. Clin. Oncol. 18 (2000) 1809-1811.

4. Dove, A. Antisense and sensibility. Nat. Biotechnol. 20 (2002) 121-124.

5. Cho-Chung, Y.S. Antisense DNAs as targeted therapeutics for cancer: No longer a dream. Curr. Opin. Invest. Drugs $\underline{3}$ (2002) 934-939. 